\title{
ENERGY EFFICIENT, LIFETIME IMPROVING AND Secure Periodic Data Collection Protocol FOR WIRELESS SENSOR NETWORKS
}

\author{
P.Anuja ${ }^{1}$ and Dr.S.Raju ${ }^{2}$ \\ ${ }^{1}$ M.E.(CSE) Student, Department of Computer Science and Engineering, \\ Sri Venkateswara College of Engineering, Pennalur, Sriperumbudur - 602 117, India \\ ${ }^{2}$ Professor, Department of Computer Science and Engineering, \\ Sri Venkateswara College of Engineering, Pennalur, Sriperumbudur - 602 117, India
}

\begin{abstract}
The most emerging prominent sensor network applications collect data from sensor nodes and monitors periodically. Resource constraint Sensor motes sense the environment and transit data to the remote sink via multiple hops. Minimum energy dissipation and secure data transmission are crucial to such applications. This paper delivers an energy efficient, lifetime improving, secure periodic Data Gathering scheme that is a hybrid of heuristic path establishment and secure data transmission. This protocol uses artificial intelligence (AI) based $A^{*}$ heuristic search algorithm to establish energy efficient admissible optimal path to sink in terms of high residual energy, minimum hop counts and high link quality. This scheme also adopts block encryption Rivest Cipher (RC6) Algorithm to secure the transmission of packets. This code and speed optimized block encryption provides confidentiality against critical data and consumes less energy for encryption. This proposed method increases the network lifetime there by reducing the total traffic load. Evaluation of performance analysis of this algorithm using Network Simulator (NS2) shows the superiority of the proposed scheme.
\end{abstract}

\section{KEYWORDS}

WSN; Data Collection; A* Heuristic Search; RC6; Security;

\section{INTRODUCTION}

Self-organizing Sensor networks dynamically changes the network topology and distributed either randomly or uniformly. A huge amount of tiny sensor nodes (SNs) monitor temperature, humidity, motions and sound. In multi-hop transmission of WSN each sensor nodes play dual characteristic of perceiving the environment and forwards the collected data to the base station (BS) via integrated radio transmitters. The key challenge is to prolong the lifetime of WSN since it is not possible to recharge the batteries of SNs in unattended environment. Considering every node in the network for a time periodic data collection generates more traffic. So the period for data collection is to be enough for collecting data from nodes. To avoid traffic congestion and packet drop over transmission only random nodes to be selected for data collection in every miniature period. Therefore, energy efficient mechanisms are required for computation operations like data storage, path construction and decision making of source nodes and to secure the communication from sources to sink. 


\subsection{Multihop Communication}

Communication in WSNs is application-dependent and also depends on the time criticality of the data. Communication can be occurred on behalf of time-driven, event-driven, query-driven and hybrid approach. The major Data collection techniques involved in WSN are 1.Tree Based data collection, 2.Hierarchical Data collection, and 3.Chain Based Data collection.

In typical wireless multi-hop networks (Fig.1.1), nodes cooperate with each other by forwarding or relaying each other's packets, possibly involving many intermediate relay nodes. This enables nodes that cannot hear each other directly to communicate over intermediate relays without increasing transmission energy.

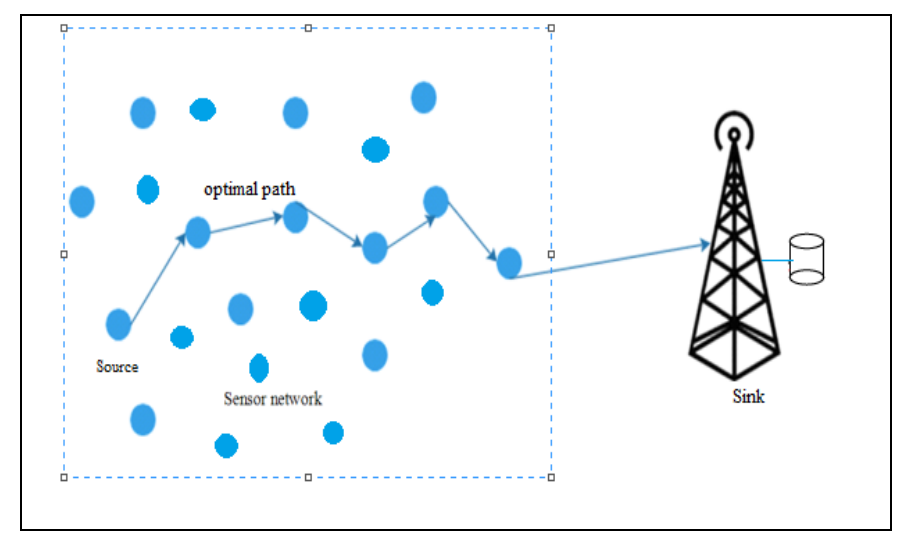

Figure 1.1. Mulihop Data Communication

Such multi-hop relaying is a very promising solution for increasing throughput and providing coverage for a large physical area. By using several intermediate nodes, the sender can reduce transmission energy by efficiently using network bandwidth. In sensor networks, the medium is shared and nodes arrange access to the medium in a distributed way independent of their current traffic demand. In particular given standard ad-hoc routing protocols that try to minimize relaying nodes on the path, nodes closer to the network centre are more likely to become a relay node. This has the inherent drawback that a node that serves as a relay node for transmissions of multiple neighbouring nodes is prone to become a performance bottleneck. As it is necessary to understand performance of such relay networks, multiple relays are involved across an end-to-end path, it is important to control performance overhead for each single packet transmission. In the process of data acquisition, the message dissemination may be subject to security issues like eavesdropping, malicious routing, and message tampering.

\subsection{Symmetric key cryptographic WSN}

The security services in WSNs are usually centred on cryptography. The four main goals of cryptography are confidentiality, integrity, authentication and non-repudiation. However, due to the constraints in WSNs, most of the existing secure algorithms cannot be directly used in WSNs. Cryptographic algorithms ensure the security of sensor network by encryption methods. In the context of cryptography the information to be sent is known as plain text. This plain text is converted to cipher text by exemption. An authentic receiver can decrypt the cipher text back into plain text by the process of decryption. The encryption and decryption use keys to process. The two vital types of cryptographic techniques are: symmetric key algorithm and asymmetric key algorithm. Symmetric key algorithm uses the same key for encryption and decryption while the asymmetric key symmetric encryption algorithm uses two different keys as pair for encryption and decryption. Due to constraint computing power and storage space of sensor nodes, 
asymmetric encryption algorithms have computational complexity and energy consumption makes it difficult to be applied to wireless sensor networks. Symmetric encryption algorithm is widely used in wireless sensor networks because of its simple calculation and small amount of calculation.

Selecting the most appropriate cryptographic method is most important in WSN design because the strength of the security system is ensured through it. The selected cryptographic algorithm should be of low code size, low data size, less processing time and low power consumption. Symmetric key cryptography is superior to public key cryptography in terms of speed, low data size and low energy cost. Hence, we use the Rivest Cipher 6 block encryption for measurement packet message.

\section{Routing Protocols}

The objective of routing is to forward data from a sender to one or more destinations. Particularly, Routing in wireless multi-hop network is a challenging task. Path establishment based routing protocols in sensor networks are usually divided into proactive, reactive and hybrid routing. A proactive protocol evaluates routes to all reachable nodes and attempt to maintain consistent upon relevant data routing information. In a reactive protocol, routing paths are searched only when needed. Hybrid protocols combine proactive routing with reactive routing in hierarchical network structures. Almost of network based routing protocols are categorized as Flat Routing, Hierarchical Routing or Location-based Routing. In flat routing technique in which all the sensor nodes play the same roles, such as collecting data and communicating with the sink, i.e. all the data collected in the remote area can be same or duplicated as all the sensor nodes work in the same way. In hierarchical routing technique all the routing sensors in the network are clustered and a cluster head collects and aggregates the data and checks for redundancy of the data that is collected before it is sent to the sink. This saves communication processing time and also saves energy dissipation. In location-based routing, all the sensor nodes are addressed by using their locations. Depending upon the strength of the incoming signals, it is possible to calculate the nearest neighbouring node's distance. As energy is the major factor of concern in routing protocols, location-based schemes demand that nodes should change their state from active to sleep mode when there is no activity. While more nodes in sleep mode, the more energy is saved. Hence, we use $A^{*}$ Heuristic search based on distance plus cost and the high link quality between nodes towards the sink.

\section{RELATED WORK}

Junyoung Heo et al [1] have introduced a novel algorithm for packet forwarding; it randomly selects the next node. A path with lower energy cost is likely to be selected, because the probability is inversely proportional to the energy cost to the sink node. To achieve real-time delivery, only paths that may deliver a packet in time are selected. However it does not ensures the traffic congestion and network lifetime.

Guangsong Yang et al [2] have proposed compressed sensing based data collection method. It considers the sparse representation of nodes in a matrix and revised isometric property of $11 \mathrm{norm}$ minimization for path construction. Even though it uses the energy efficiency and improves lifetime of network it does not provide secure transmission of packets.

Chao Wang, Huadong Ma et al [3] have focused on approximation in data collection. It estimates local approximation at cluster head and global approximation at sink. Also it uses greedy heuristic algorithm to provide the approximation within the subset of nodes. 
Learning from predefined approximated values cannot predict the nearest absolute and admissible path until it reaches the sink.

Lifetime is another constraint area that has been extensively studied in WSNs. Zhang et al [4] investigated the unbalanced energy consumption for uniformly deployed data gathering sensor networks. In this paper, the network is divided into multiple corona zones and each node can perform data aggregation. A localized zone-based routing scheme was proposed to balance energy consumption among nodes within each corona. Liu et al. in [5] formulated the integrated design of route selection, traffic load allocation, and sleep scheduling to maximize the network lifetime. Based on the concept of opportunistic routing, [6] developed a routing metric to address both link reliability and node residual energy. The sensor node computes the optimal metric value in a localized area to achieve both reliability and lifetime maximization.

Yanjun Yao et al [7] correlated the data collection of WSN with open vehicular routing and provided heuristic algorithms to improve the accuracy and to reduce latency of data. However, it does not ensure about data confidentiality and channel eavesdropping.

\section{SeCURITy ANALYSIS}

By consider the major concern as security; we analyze some specific parameters of performance among various symmetric key encryption algorithms and finally we decided to choose the RC6 due to its superiority than other algorithms. The following table illustrates the analysis of security against various algorithms.

Table I - Security Analysis of Algorithms

\begin{tabular}{|l|l|l|l|l|}
\hline $\begin{array}{l}\text { Performance } \\
\text { parameters }\end{array}$ & \multicolumn{4}{|c|}{ Algorithm } \\
\hline & DES & $\begin{array}{c}\text { TWO } \\
\text { FISH }\end{array}$ & \multicolumn{1}{|c|}{ AES } & RC6 \\
\hline Throughput & Very low & High & High & $\begin{array}{l}\text { Very } \\
\text { high }\end{array}$ \\
\hline $\begin{array}{l}\text { Execution } \\
\text { Time }\end{array}$ & Very high & Low & Moderate & Very low \\
\hline $\begin{array}{l}\text { Cryptanalysis } \\
\text { (linear \& } \\
\text { Differential) }\end{array}$ & Easy & Difficult & Difficult & $\begin{array}{l}\text { Very } \\
\text { Difficult }\end{array}$ \\
\hline Rounds & 16 & 16 & $12,14,16$ & 20 \\
\hline Key Size & 56 & $32-448$ & $\begin{array}{l}128,192, \\
256\end{array}$ & $\begin{array}{l}128,192, \\
256\end{array}$ \\
\hline Security & In Secure & High & High & $\begin{array}{l}\text { Very } \\
\text { High }\end{array}$ \\
\hline
\end{tabular}




\section{SYSTEM ARCHITECTURE}

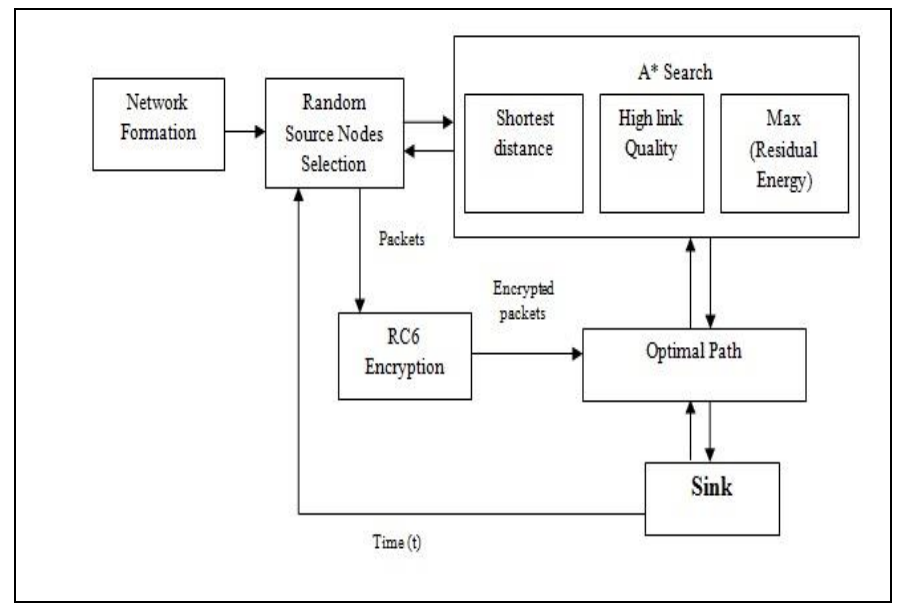

Figure 5.1. System Architecture

This proposed system architecture shows an innovative data gathering method in accordance with energy efficiency and network lifetime of the sensor field. Multi hop data routing towards sink helps to improve the data loss due to long communication range. In which the sink selects random number of nodes periodically that is for every period of time to collect information stored on those nodes. The optimal shortest path is established and the routing table is maintained at sink. The path considers the sensor parameters such as shortest distance, remaining energy level of nodes and the high link quality for optimization. Each source nodes send its encrypted data via optimal path. The remote sink collects the encrypted data and decrypted it at sink to maintain confidentiality of data packets.

\section{Modules Description}

1. INITIAL STAGE

2. RANDOM NODE SELECTION

3. OPTIMAL PATH CONSTRUCTION

4. SECURE TRANSMISSION

5. DATA GATHERING

\section{INITIAL STAGE}

At initial stage, for start of every time period $\mathrm{M}$ out of $\mathrm{N}$ random nodes is selected as source nodes from the sensor network field. Each node knows the location of sink (Base station) and also by its own. Every node in the network sends its energy level to its neighbour within the communication range and to the sink. The node which has minimum energy level than the threshold cannot participate in the data forwarding.

\section{RANDOM NODE SELECTION}

For each period of time t, the sink selects a set of random nodes as source nodes and collects the encrypted data from those nodes via optimal path. The sink selects $\frac{m}{N}$ nodes as sources from the network of sensor field. 


\section{Optimal Path Construction}

A* Heuristic Search algorithm is used to construct optimal path from each sources to sink. It considers the shortest distance, Maximum Residual Energy, High link Quality node as its nexthop from the neighbour list. The following data flow diagram depicts the optimal path establishment from each source to base station based on the service parameters of sensor nodes.

A* algorithm uses the heuristic function $f(n)=h(n)+g(n)$, where

$$
g(n)=\max \left\{\frac{E_{\text {res }}(n)}{E_{\text {ini }}(n)}+l_{q}(n)\right\}
$$

Where, $\frac{E_{\text {rgs }}(n)}{E_{\text {ini }}(n)}$ is the current energy level of node $\mathrm{n}$,

$l_{q}(n)$ is the link quality of node $\mathrm{n}$ that is inversely proportional to the link weight $\mathrm{w}$, (ie)

$l_{q}(n)=\frac{1}{w(n, j)}$

where $\mathrm{j}$ is the one hop neighbour nodes, and

$h(n)=\frac{1}{\min \left(h c_{n}^{g}\right)}$

where, $h c_{n}^{s}$ is the minimum hop count from node $\mathrm{n}$ to destination $\mathrm{s}$, that is the fraction of distance between node $\mathrm{n}$ to $\mathrm{s}$ and the average distance between node $\mathrm{n}$ to one hop neighbour $\operatorname{list}(\mathrm{j})$.

To calculate the distance between node and sink node via Euclidean distance formula as,

$$
\mathrm{d}(\mathrm{n}, \mathrm{s})=\sqrt{\left(\mathrm{x}_{\mathrm{n}}-\mathrm{x}_{\mathrm{s}}\right)^{2}+\mathrm{b}\left(\mathrm{y}_{\mathrm{n}}-\mathrm{y}_{\mathrm{s}}\right)^{2}}
$$

Where, ${ }^{\mathrm{d}(\mathrm{n}, \mathrm{s})}$ is equal to the Euclidian distance between the node $\mathrm{n}$ and sink node s.

The following data flow diagram in figure 7.1. illustrates the optimal path construction between each randomly selected node to the sink node. 


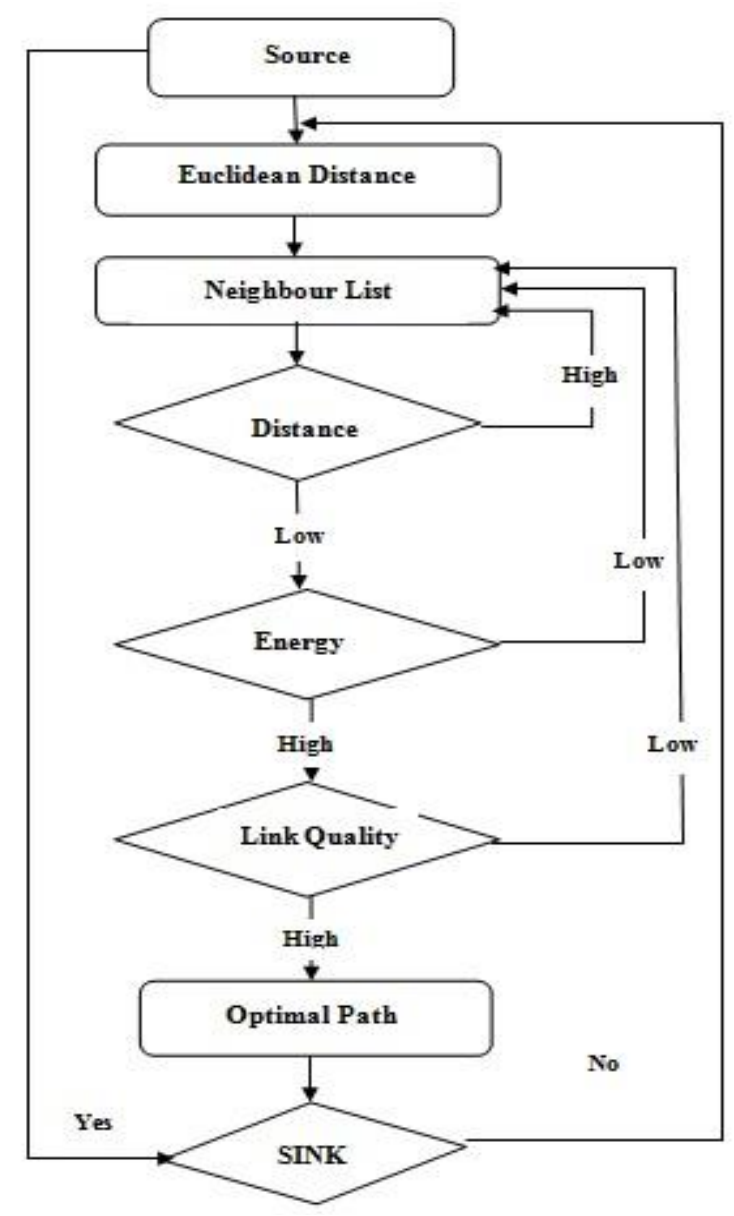

Figure 6.1. DFD of Optimal path Establishment

\section{SECURE TranSMISSION}

To avoid channel eavesdropping and to provide confidentiality against the attacker, each packets from the sources are encrypted using RC6 block encryption. It uses pre whitening and post whitening. Pre whitening is to avoid the reveal of plaintext and post whitening is to prevent the reveal of cipher text. The 20 rounds RC6 is secure against both linear and differential crypt analysis. RC6 requires least space to store its code in cache memory. Only the decryption takes more time than encryption, hence the resource limited source node involves only in encryption and the high processing sink node decrypt the data in offline at the end of each period of data collection. The following figure 7.2 shows the 20 rounds RC6 advanced encryption standard work flow. 


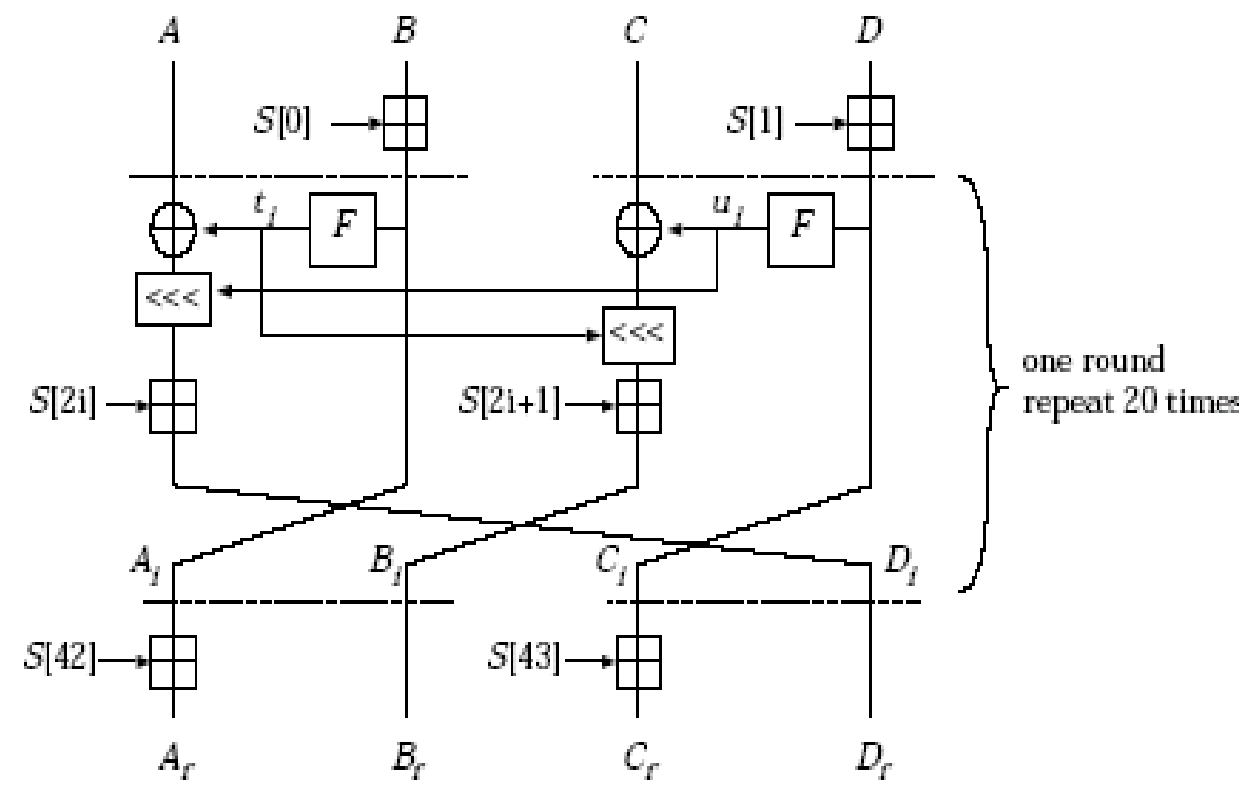

Figure 6.2. RC6 Encryption

\section{DAta Gathering}

The sink broadcasts the encryption signal to the sources after path construction. Each source node encrypts the data using with the expanded key from source node after mutual authentication. The sink/Base station decrypt the data received from sources and stored it for future purpose. The decryption is the reverse process of encryption. Since the decryption is done in offline the energy consumption is reduced.

\section{RESULTS}

Hence, the energy usage for data encryption and transmission over the optimal path is very low when compared with MST and LRR baseline protocols. The shortest path routing using dijikstra's algorithm also consumes much energy and involves more nodes for transmission. The lifetime of the network is prolonged by the proposed work since it uses the nodes above the energy threshold value for data transmission. The probabilistic random nodes are selected for every time period is shown as colored nodes helps to reduce traffic congestion. Immediately after selection of random sources, it triggers the $\mathrm{A}^{*}$ heuristic for optimal path construction and RC6 for encryption and the encrypted data transmission over that energy efficient optimal path to source to sink is mentioned as blocked square boxes.

The result analysis of the proposed protocol using Network Simulator2.35(NS2) is shown further. 
International Journal on Computational Science \& Applications (IJCSA) Vol.6, No. 3, June 2016

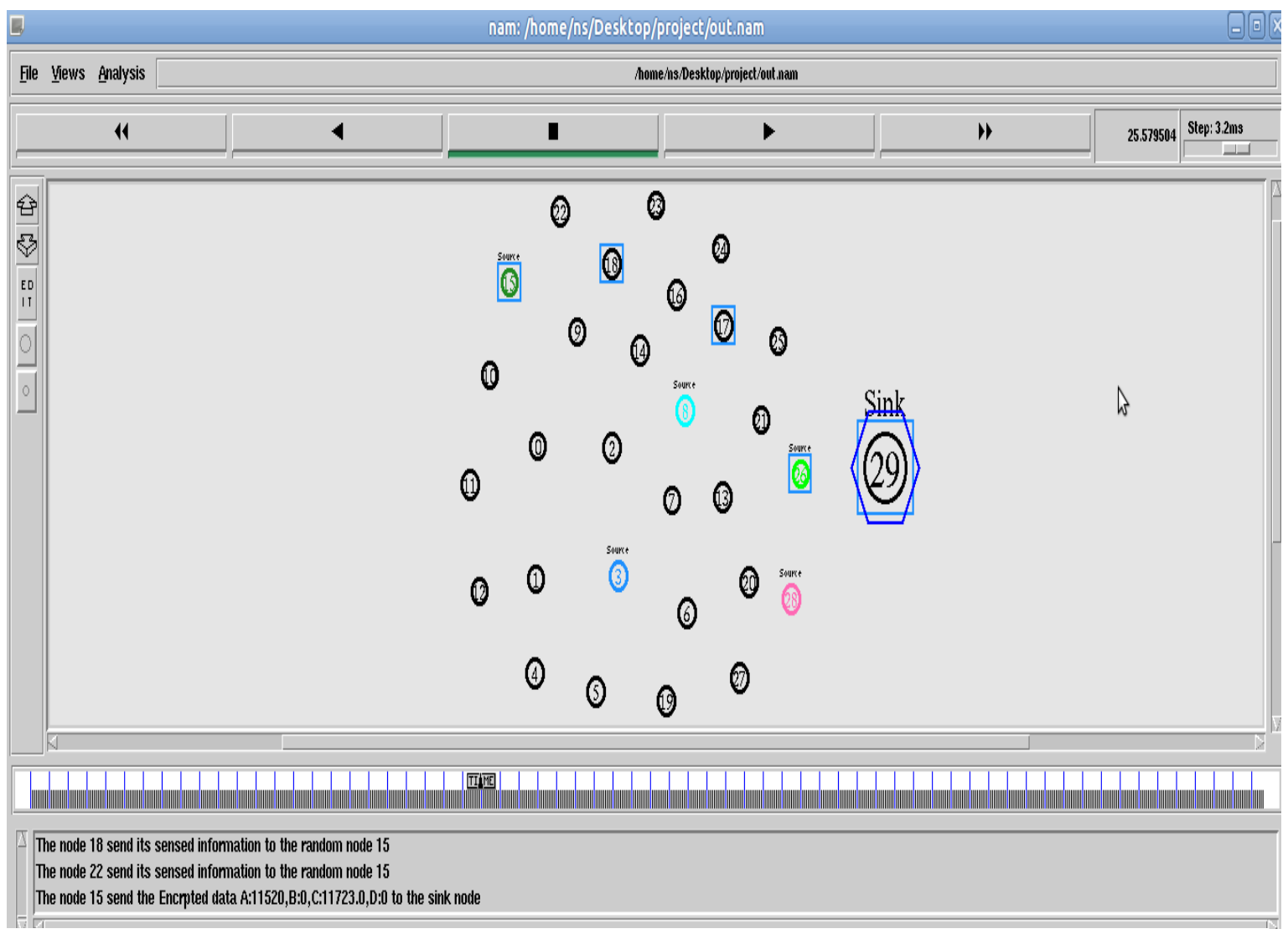

Figure 7.1. Energy Efficient Optimal path

\section{REMAINING ENERGY LEVEL}

The current energy level is the percentage of residual energy from its initial energy. Also it clearly shows the network lifetime metric is maintained after data transmission. That is the time to occur the first dead node is the time of topology changes in the network. Because of energy efficient routing the lifetime of network is maintained.

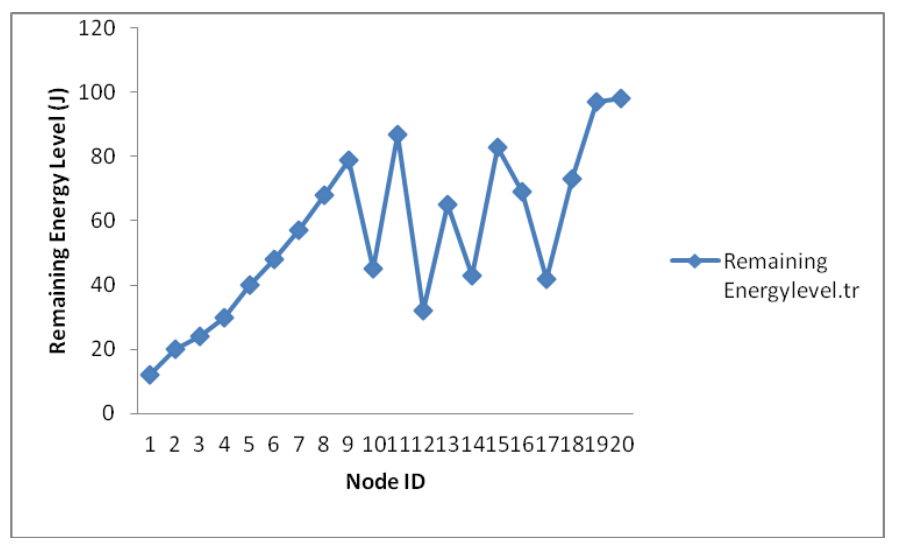

7.2. Remaining Energy Level of nodes in the network 


\section{ENERGY CONSUMPTION OF NODE}

The energy consumption of each node is the energy required to receive and/or transmit packets via that node.

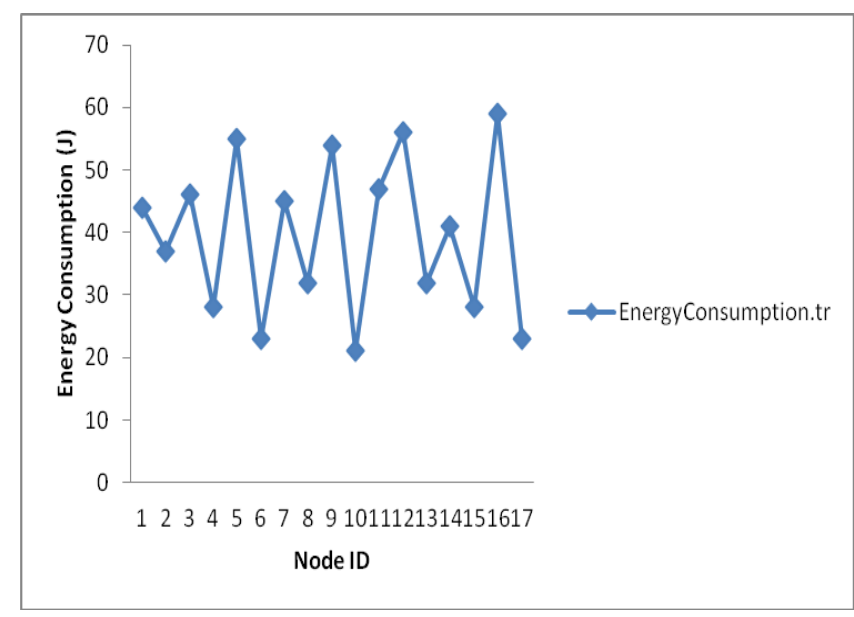

7.3. Energy Consumption of individual nodes

\section{TOTAL ENERgY CONSUMPTION}

Total energy consumption is calculated as energy consumed by the nodes which involved in entire transmission from randomly selected sources to sink. The total energy consumption is computed by using the following formula

$\mathrm{TE}=\sum_{\mathrm{i}}^{\mathrm{l}} \mathrm{EC}_{\mathrm{i}}$

Where $1=$ number of links Eci=Energy consumption of ith node.

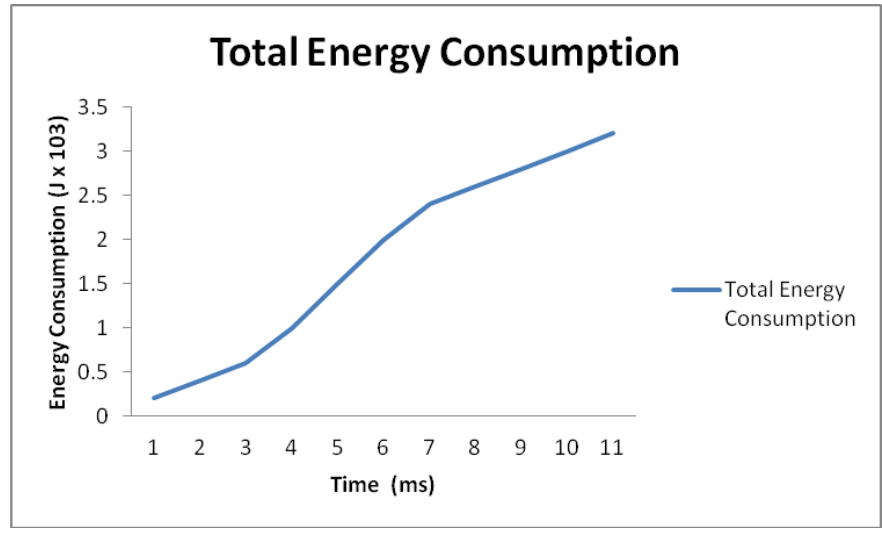

7.4. Total Energy Consumption for a time period 


\section{CONCLuSion}

In this paper, the main contribution of our work is focused on the energy efficiency, security and lifetime of the network. It increases the packet delivery ratio from source to sink via optimal path by reducing the receipt of packet delay over transmission. The lifetime of the deployed sensor network is also improved by assigning weights to links based on the remaining energy level of individual nodes. There is no packet drop via optimal path since it is generated dynamically. The simulation results show the performance superiority of the proposed scheme.

\section{REFERENCES}

[1] Junyoung Heo, Jiman Hong, and Yookun Cho, Member, IEEE, "EARQ: Energy Aware Routing for Real-Time and Reliable Communication in Wireless Industrial Sensor Networks",'IEEE TRANSACTIONS ON INDUSTRIAL INFORMATICS, VOL. 5, NO. 1, FEBRUARY 2009.

[2] Guangsong Yang, Mingbo Xiao, and Shuqin Zhang, School of Information Technology, Jimei University, Xiamen, China "Data Aggregation Scheme based on Compressed Sensing in Wireless Sensor Network", JOURNAL OF NETWORKS, VOL. 8, NO. 1, JANUARY 2013.

[3] Chao Wang, Huadong Ma, Member, IEEE, Yuan He, Member, IEEE, and Shuguagn Xiong, "Adaptive Approximate Data collection for Wireless Sensor Networks", IEEE TRANSACTIONS ON PARALLEL AND DISTRIBUTED SYSTEMS, VOL. 23, NO. 6, JUNE 2012.

[4] H. Zhang and H. Shen, "Balancing energy consumption to maximize network lifetime in datagathering sensor networks," IEEE Trans. Parallel Distrib. Syst., vol. 20, no. 10, pp. 1526-1539, Oct. 2009.

[5] F. Liu, C.-Y. Tsui, and Y. J. Zhang, "Joint routing and sleep scheduling for lifetime maximization of wireless sensor networks," IEEE Trans. Wireless Commun., vol. 9, no. 7, pp. 2258-2267, Jul.2010.

[6] C.-C. Hung, K.-J. Lin, C.-C. Hsu, C.-F. Chou, and C.-J. Tu, "On enhancing network-lifetime using opportunistic routing in wireless sensor networks," in Proc. 19th Int. Conf. Comput. Commun.Netw., Aug. 2010, pp. 1-6.

[7] Yanjun Yao, Qing Cao, Member, IEEE, ACM, and Athanasios V. Vasilakos, Senior Member, IEEE," EDAL: An Energy-Efficient, Delay-Aware, and Lifetime-Balancing Data Collection Protocol for Heterogeneous Wireless Sensor Networks", IEEE/ACM TRANSACTIONS ON NETWORKING, VOL. 23, NO. 3, JUNE 2015

[8] P. E. Hart, N. J. Nilsson, and B. Raphael, "A formal basis for the heuristic determination of minimum cost paths," IEEE Transactions on Systems Science and Cybernetics, vol. 4, no. 2, pp. 100- 107, 1968.

[9] S. Russell and P. Norving, Artificial Intelligence: A Modern Approach, 2/E, Pearson Prentice Hall.

[10] W. R. Heinzelman, A. Chandrakasan, and H.Balakrishnan, "Energy-efficient communication protocol for wireless microsensor networks," in System Sciences, 2000. Proceedings of the 33rd Annual Hawaii.

\section{Authors}

P. Anuja, completed B.E. in 2008. Then In contract basis she was worked with ECourts Mission Mode Project. She was in charge for Tuticorin district and had the designation as System officer from 2009 to 2010 May. She is pursuing master of engineering in Sri Venkateswara College of Engineering from 2014 to till date. The area of interest of her are cloud computing and network Security.

Dr. S. Raju, worked as Lecturer for 10 years, Assistant professor for 5 worked as Lecturer for 10 years, Assistant professor for 5 Years, Associate Professor for 4 years and he worked as Professor from 2014 to 2016th May. Now he is currently working in AMITY Global Business School \& AMITY Institute of Higher Education, Mauritius as Professor and Head.
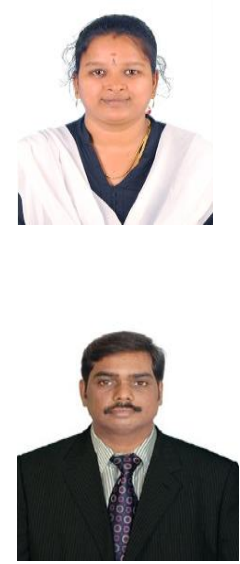